\title{
Dental Stem Cells- Potential for Tooth Regeneration
}

\author{
Ruby Dwivedi* and Shilpa Trivedi \\ Department of Oral and Maxillofacial Surgery, King George's Medical University, India
}

Submission: August 25, 2020; Published: November 02, 2020

*Corresponding author: Ruby Dwivedi, Research Associate, Department of Oral and Maxillofacial Surgery, King George's Medical University, Shahmina Road, Chowk, Lucknow-226030, Uttar Pradesh, India

\section{Abstract}

Dental Stem cells are undifferentiated cells isolated from tooth and associated structures. Dental stem cells can be easily obtained from both adult and deciduous teeth, the periodontal ligament, the apical papilla and the dental follicle. Their subsequent differentiation can be utilized to replace the lost tooth structures. Here we aim to highlight potential of dental stem cells from varied origin for tooth regeneration. Several studies have demonstrated tooth tissue regeneration. Application of recent 3D bioprinting technology has further enhanced probability of accurate tooth tissue regeneration. However complete tooth regeneration is still a future thing.

Keywords: Dental stem cells

Abbreviations: SHED: Stem Cells from Human Exfoliated Deciduous Teeth; DPSC: Dental Pulp Stem Cells; SCAP: Stem Cells from Apical Papilla; PDLSC: Periodontal Ligament Derived Stem Cells; DFPC: Dental Follicle Precursor Cells

\section{Introduction}

Mesenchymal stem cells (MSCs) are the unspecialized cells that have the capacity to differentiate into multiple specialized cell types, each having new specialized cell functions. Though adult stem cells are not totipotent like embryonic stem cells, they have proliferative, differentiating and regenerative potential. There is great potential for the isolation of MSCs from a single tooth that may have a wide range of uses. Stem cells have been obtained from the dental pulp of both adult and deciduous teeth, the periodontal ligament, the apical papilla and the dental follicle. Dental stem cells are obtained relatively easily, thus making them an appropriate source for autologous stem cell transplantation to replace lost tooth structures.

Repair of dental pulp and periodontal ligament is a huge clinical challenge as human teeth exhibit very limited regeneration capacity. Stem cells may be useful for restoring vital pulp tissue lost because of infection, periodontal tissues damaged due to periodontal diseases and for reestablishment of lost partial or complete tooth structures to form biological implant. These cells may be used for dentinal repair of many teeth. This mini review aims to highlight potential of dental stem cells from varied origin for tooth regeneration.

\section{Dental stem cells: types and regenerative potential}

Gronthos et al. [1] isolated stem cells from dental pulp of impacted third molars and it was termed dental pulp stem cells (DPSC). These cells have a high proliferation rate and the ability to sustain self- renewal. DPSCs when transplanted in immunocompromised mice demonstrated the ability to form functional dental tissue in the form of pulp- dentin complex. 1 Further study demonstrated that DPSCs were also capable of differentiating into other mesenchymal cell derivatives in vitro such as odontoblasts, adipocytes, chondrocytes and osteoblasts $[2,3]$. It has been documented that stem cells isolated from the pulp of human exfoliated deciduous teeth (SHED) can generate dentine, inducing bone formation and differentiation into other non-dental mesenchymal cell derivatives in vitro $[4,5]$.

SHED and other dental stem cells are derived from neural crest ectomesenchyme, and so may appear developmentally identical but studies have shown that there are differences. Compared to DPSCs, SHED have higher proliferation rates, increased population doublings and osteoinductive capacity in vivo [6]. In a study, SHED was seeded onto tooth slices/ scaffolds and implanted subcutaneously into immunodeficient mice, it was found that SHED 
differentiated into functional odontoblasts capable of generating tubular dentine and angiogenic endothelial cells [7]. In another study, combining SHED with injectable scaffolds (Puramatrix ${ }^{\mathrm{TM}}$ and rh Collagen type I) revealed appreciable survival of dental stem cells when injected into human premolar root canals in vivo, complete reconstruction of vascularized pulp tissue throughout the root canal, and differentiation to DSPP and DMP-expressing odontoblasts responsible for generation of new dentin [8].

Stem cells from apical papilla (SCAP) are characterized by a high proliferation rate that makes them suitable for forming odontoblast-like cells for a cell-based regeneration, being able to produce dentin in vivo and to complete the root formation. SCAP is an important cell source for pulp regeneration as the apical papilla can survive even during the process of cell necrosis. SCAP can differentiate into odontoblasts and adipocytes and SCAP exhibit higher proliferation rates than DPSC [9]. Huang et al. [10] demonstrated that the in vivo implantation of SCAP with a scaffold allowed the formation of pulp-like tissues in the root canal. The formation of heterotopic dental pulp/dentin complex in empty root canals using SCAP-cell sheet-derived pellet even without a scaffold has been reported by another study [11].

In immuno-compromised mice, using ex vivo-expanded human DPSCs and SCAP, synthetic scaffolds and human root segments, the regeneration of vascularized pulp-like tissue and the formation of dentin-like mineral structures depositing onto the existing dentinal wall in the root canal space was demonstrated. 10 It was found that the root canal space was entirely filled by a pulp-like tissue with well-established vascularity. Furthermore, along the canal dentinal wall, deposition of a continuous layer of dentin-like tissue was observed.

The stem cells from human periodontal ligament (PDL) are capable of differentiation along mesenchymal cell lineages to produce cement oblast-like cells, adipocytes, and connective tissue rich in collagen I [13]. The cell surface marker characteristics and differentiation potential of periodontal ligament stem cells (PDLSC) has been displayed to be comparable to bone marrow stromal stem cells and DPSC [13]. Transplantation of PDLSC into immuno-compromised mice, resulted in formation of cementum/ PDL-like structures.

Co- transplantation of both SCAP (to form the root) and PDLSC (to from the periodontal ligament) into tooth sockets of mini pigs model resulted in generation of a root/ periodontal complex which was competent of supporting a porcelain crown thus restoring normal tooth function [9]. This suggests that these cell populations can be used to generate biological roots like metal implants, by capping with artificial crowns. The dental follicle is considered a multipotent tissue, as it has the capacity to generate cementum, bone and PDL from the ectomesenchyme-derived fibrous tissue. Dental follicle precursor cells (DFPC) have also been isolated and grown under defined tissue culture conditions.
More recently, characterization of these stem cells has exhibited their potential for use in tissue engineering applications, including periodontal and bone regeneration [14].

A novel approach for clinical translation of these biology- based studies would be the endogenous recruitment of progenitor/ stem cells into the root canals for dental pulp regeneration. Advances are being made to develop and replace entire tooth in adult environment thus achieving complete tooth regeneration.

\section{Current trend: 3D bio-printed dental tissue regeneration using dental stem cells}

In this context, modern 3D bioprinting modalities have imperatively demonstrated tooth regeneration using extracellular matrix (ECM) derived scaffolds and cytocompatibility microenvironments for tissue regeneration. Athirasala et al. [15] developed a novel bioink, blending printable alginate $(3 \% \mathrm{w} / \mathrm{v})$ hydrogels with fractions of the dentin matrix. They demonstrated odontogenic differentiation of stem cells from the apical papilla encapsulated in bioprinted hydrogels [15]. Although several advances have been made in dental tissue regeneration using various 3D printed scaffolds of different materials like gelatin/alginate hydrogels or 3D bioprinted PCL (poly-epsiloncaprolactone)/hydroxyapatite and incorporating stem cells from varied sources like human dental pulp cells (HDPCs) and apical papilla. Apparently, none of them could result in complete formation of tooth and its associated structures [16,17]. Further developments in technology and the shapes and composition of carriers for these cells make the possibility of creating a viable dental implant a reality in not too distant future.

\section{Conclusion}

Despite the advances made in dental tissue regeneration employing stem cells from varied sources, tooth regeneration is still a future thing. The chief limiting factor is that majority of the studies were done on small animal models so they cannot be directly translated to humans in the clinics. Nevertheless, it would not be an overstatement to say that stem cell based regenerative procedures are the future of dentistry..

\section{References}

1. Gronthos S, Mankani M, Brahim J, Robey PG, Shi S (2000) Postnatal human dental pulp stem cells (DPSCs) in vitro and in vivo. Proc Natl Acad Sci USA 97: 13625-13630.

2. Koyama N, Okubo Y, Nakao K, Bessho K (2009) Evaluation of pluripotency in human dental pulp cells. J Oral Maxillofac Surg 67: 501-506.

3. Yu J, He H, Tang C, Zhang G, Li Y, et al. (2010) Differentiation potential of STRO-1+ dental pulp stem cells changes during cell passaging. BMC Cell Biol 11: 32 .

4. Miura M, Gronthos S, Zhao M, Lu B, Fisher LW, et al. (2003) SHED: stem cells from human exfoliated deciduous teeth. Proc Natl Acad Sci USA 100: 5807-5812. 
5. Shi S, Bartold PM, Miura M, Seo BM, Robey PG, et al. (2005) The efficacy of mesenchymal stem cells to regenerate and repair dental structures. Orthod Craniofac Res 8: 191-199.

6. Nakamura S, Yamada Y, Katagiri W, Sugito T, Ito K, et al. (2009) Stem cell proliferation pathways comparison between human exfoliated deciduous teeth and dental pulp stem cells by gene expression profile from promising dental pulp. J Endod 35: 1536-1542.

7. Sakai VT, Zhang Z, Dong Z, Neiva KG, Machado MA, et al. (2010) SHED differentiate into functional odontoblasts and endothelium. J Dent Res 89: 791-796.

8. Rosa V, Zhang Z, Grande RH, Nör JE (2013) Dental pulp tissue engineering in full-length human root canals. J Dent Res 92: 970-975.

9. Sonoyama W, Liu Y, Fang D, Yamaza T, Seo BM, et al. (2006) Mesenchymal stem cell-mediated functional tooth regeneration in swine. PLoS One 1: e79.

10. Huang GT, Yamaza T, Shea LD, Djouad F, Kuhn NZ, et al. (2010) Stem/ progenitor cell-mediated denovo regeneration of dental pulp with newly deposited continuous layer of dentin in an in vivo model. Tissue Eng Part A 16: 605-615.

11. Na S, Zhang H, Huang F, Wang W, Ding Y, et al. (2016) Regeneration of dental pulp/ dentine complex with a three-dimensional and scaffoldfree stem-cell sheet-derived pellet. J Tissue Eng Regen Med 10(3): 261270 .

12. Seo BM, Miura M, Gronthos S, Bartold PM, Batouli S, et al. (2004) Investigation of multipotent postnatal stem cells from human periodontal ligament. Lancet 364: 149-155.

13. Lin NH, Gronthos S, Mark Bartold P (2009) Stem cells and future periodontal regeneration. Periodontol 51: 239-251.

14. Morsczeck C, Götz W, Schierholz J, Zeilhofer F, Kühn U, et al. (2005) Isolation of precursor cells (PCs) from human dental follicle of wisdom teeth. Matrix Biol 24(2): 155-165.

15. Athirasala A, Tahayeri A, Thrivikraman G, França CM, Monteiro N, et al. (2018) A Dentin-Derived Hydrogel Bioink for 3D Bioprinting of Cell Laden Scaffolds for Regenerative Dentistry. Biofabrication 10(2): 024101.

16. Liu J, Ruan J, Weir MD, Ren K, Schneider A, et al. (2019) Periodontal Bone-Ligament-Cementum Regeneration via Scaffolds and Stem Cells. Cells 8(6): 537.

17. Kim K, Lee CH, Kim BK, Mao JJ (2010) Anatomically Shaped Tooth and Periodontal Regeneration by Cell Homing. J Dent Res 89(8): 842-847.

\section{Your next submission with Juniper Publishers will reach you the below assets}

- Quality Editorial service

- Swift Peer Review

- Reprints availability

- E-prints Service

- Manuscript Podcast for convenient understanding

- Global attainment for your research

- Manuscript accessibility in different formats

( Pdf, E-pub, Full Text, Audio)

- Unceasing customer service

Track the below URL for one-step submission https://juniperpublishers.com/online-submission.php 
